\title{
Short communication: Progression of Johne's disease curtailed by a probiotic
}

\author{
R. E. Click ${ }^{1,2}$ and C. L. Van Kampen ${ }^{3}$ \\ University of Wisconsin-River Falls, River Falls 54022
}

\begin{abstract}
The naturally occurring inflammatory bowel disease Johne's, caused by Mycobacterium avium ssp. paratuberculosis (MAP), has many clinical manifestations in common with the human inflammatory bowel disease Crohn's disease. In addition, both lack preventive and curative therapies. Because a high percentage of Crohn's patients harbor MAP, it is not surprising that MAP is at the center of controversy as to its contribution. Special concern is being raised as to what role, if any, food animals play in transmission of MAP to humans. Because management practices, presently considered the best way to control the spread of MAP, have not and most likely will not eliminate MAP from food animals, other preventive or curative measures are needed. The results presented herein show that a unique bacterium, Dietzia ssp. C79793-74, used as a probiotic, was therapeutic for adult paratuberculosis animals, and resulted in a cure rate of $37.5 \%$.
\end{abstract}

Key words: Johne's disease, probiotic, Dietzia, inflammatory bowel disease

The naturally occurring inflammatory bowel disease (IBD) found predominantly in ruminants called Johne's disease is caused by Mycobacterium avium ssp. paratuberculosis (MAP). For paratuberculosis to be manifested in cattle, both infection with MAP and inflammation of the intestine are required. Infection that results in disease can occur via any of the following, primarily at the neonatal or early postnatal stage: 1) in utero (Seitz et al., 1989; Sweeney et al., 1992a; Whittington and Windsor, 2009), 2) colostrum or milk (Taylor et al., 1981; Sweeney et al., 1992b; Streeter et al., 1995), and 3) ingestion of fecal contaminated material (Chiodini et al., 1984; Sweeney, 1996). Interestingly, Johne's disease has many manifestations in common with a human subtype IBD, Crohn's disease,

\footnotetext{
Received February 14, 2009.

Accepted June 30, 2009.

${ }^{1}$ Corresponding author: mbclicker@msn.com

${ }^{2}$ Retired; current address: N8693 1250 Street, River Falls, WI 54022 .

${ }^{3}$ Current address: Excorp Medical Inc., Minneapolis, MN.
}

including the most clinically notable, debilitating diarrhea (Chiodini, 1989; Clarke, 1997; Scanu et al., 2007). Because a high percentage of Crohn's patients, relative to the general population, are infected systemically with MAP, it not surprising that MAP is at the center of controversy with regard to its role in this disease (Chiodini, 1989; Clarke, 1997; Hermon-Taylor et al., 2000; Chamberlin et al., 2001; Harris and Lammerding, 2001; Hermon-Taylor and Bull, 2002; Chamberlin and Naser, 2006; Feller et al., 2007; Scanu et al., 2007; Abubakar et al., 2008; Behr and Kapur, 2008).

As Johne's disease has become an increasingly worldwide problem, due in part to the absence of a preventive vaccine or drug or curative treatment, there is concern as to what role, if any, animals play in the transmission of MAP to humans (McDowell and McElvaine, 1997; Grant et al., 2002; Ayele et al., 2005; Ellingson et al., 2005; Pickup et al., 2006; Abubakar et al., 2007; Antognoli et al., 2008). Presently, sound management practices and stringent culling are considered the best means to reduce the spread of MAP from animal to animal, as well as from farm to farm (Wells and Wagner, 2000; Kennedy and Benedictus, 2001; McKenna et al., 2006; Benedictus et al., 2008; Lu et al., 2008; Tavornpanich et al., 2008). However, because such practices have yet to (and most likely will not) completely eliminate MAP from food animals, other preventive or curative measures are needed. The research presented herein was undertaken to assess whether the bacterium Dietzia ssp. C79793-74, reported to inhibit growth of MAP under specific in vitro conditions (Richards, 1988), would have any therapeutic benefit as a probiotic for adult paratuberculosis dairy cows.

Animals for the present experiments included adult dairy cows that (a) tested negative for all parameters throughout their lifetime or (b) were at an early stage of Johne's disease as defined by clinical evaluation, serum agar gel immunodiffusion (AGID) negativity, low or negative fecal shedding, and low positive ELISA values of 1.5 to 2.5. Our final classification of an animal as positive was based on whether she tested both ELISA-positive and positive, but not necessarily concurrently, for one of the following parameters - fecal shedding, AGID, end-stage disease - or tested ELISA 
positive multiple times. An emaciated animal was defined as having end-stage disease based on the presence of "pipestream" diarrhea and depressed appetite. As an additional means to document Johne's disease of specific animals, complete pathological postmortem analysis and culture determination of MAP in tissues was done at the University of Minnesota Veterinary School Diagnostic Laboratory (St. Paul). The autopsies were designed to confirm Johne's disease status only; they were not intended to define specific aspects or extent of disease or to be compared with other antemortem parameters. Recumbent, emaciated, or cachectic endstage animals were humanely euthanized by a veterinarian when they no longer could get up and stand on their own by intravenous injection of a sodium pentobarbital solution (Fatal Plus, $6 \mathrm{~g} / \mathrm{mL}$, Vortech Pharm, Dearborn, MI). Animals that showed life-threatening ramifications from non-paratuberculosis conditions were also euthanized.

Because it was not our purpose to confirm the prognosis that Johne's-positive animals succumb to their disease, many more animals were assigned to the treated group than to the nontreated group. As positive ELISA values were detected, 1 of 4 animals was randomly assigned to the nontreated group and the other 3 to the treated group. Once a cow was enrolled in the study, the different assays were repeated at various intervals over her remaining life. All animals were housed in a tie-stall facility as a single dairy herd under field conditions designed to mimic those on a typical dairy farm. By design, the positive animals were all at an early stage of disease before initiation of treatment. The treated and nontreated positive groups were closely matched for initial ELISA values and number of lactations. The treated group comprised almost equal numbers of Holsteins and Jerseys.

Fecal material collected directly from the rectum using individual disposable gloves and blood obtained aseptically from the tail vein were transferred to sterile containers and sent overnight to Allied Monitor Inc. (Fayette, MO) for serum ELISA, serum AGID, and fecal culture analysis. The majority of fecal and serum samples were obtained concurrently. The reliability, sensitivity, and specificity of these assays, as performed by Allied Monitor, for individual animals were previously established (our unpublished data). The most important finding was that a single positive ELISA value $>1.4$, including 12 of 14 suspect values (1.5 to 2.0 ), unequivocally identified 73 out of 75 animals as Johne's positive.

Dietzia (originally classified as Mycobacterium gardonae) was isolated from fecal material of a paratuberculosis sero- and fecal-positive cow (Richards, 1988). It was reclassified as Dietzia using the gold standard for bacterial identification, DNA sequence of $16 \mathrm{~S}$ rRNA (Woese, 1987) by Midi Labs Inc. (Newark, DE). Dietzia were grown in 75-L fermenters at the University of Minnesota Biotechnology Institute for 3 to $4 \mathrm{~d}$ at $29^{\circ} \mathrm{C}$ in fructose-supplemented tryptic soy broth and then centrifuged, washed, and concentrated 20 -fold before storage in $45-\mathrm{mL}$ aliquots at $-20^{\circ} \mathrm{C}$. New lots were prepared as needed, approximately every 3 mo. Based on preliminary dosage experiments, Jerseys, Jersey $\times$ Holstein crosses, and Holsteins were treated by top dressing Dietzia on the morning feed at a daily dose of $2 \times 10^{11}, 3 \times 10^{11}$, and $4 \times 10^{11}$ cfu, respectively. If animals refused to eat, Dietzia were directly deposited into their mouths with a needleless syringe.

The basic parameters determined for the 3 different groups of animals - paratuberculosis-free, subclinical diseased animals that were not treated with Dietzia, and subclinical diseased positives that were treated with Dietzia - are shown in Table 1. Fecal shedding, ELISA, and AGID were routinely measured longitudinally over the lifetime of each animal. Also shown in the table is the number of months that each animal survived after the first positive ELISA value was detected (or after the first ELISA test for negative animals). Because several animals were terminated for health reasons unrelated to paratuberculosis, Table 1 includes a "+" sign after the number of months an animal survived to indicate that the actual survival time would have been longer.

As described by others, when ELISA values of nontreated, early-stage animals change over time, they do so by continuously increasing (Waters et al., 2003; Nielsen and Toft, 2006) and when positive change ceased, the ELISA values are reasonably stable (Nielsen et al., 2002). In contrast, fecal MAP shedding showed large variations over time (Sherman et al., 1995). Our results with Dietzia-treated animals were similar (our unpublished data). Because of 1) the lack of correlation of ELISA and MAP, 2) the high variability of MAP counts over time, and 3) the fact that only 4 of 21 initially ELISA-positive animals were also shedding MAP in feces, evaluation of any potential benefit of Dietzia treatment was based on longitudinal analysis of ELISA values. Shedding of MAP, positive AGID, appearance of clinical disease, and autopsy were parameters used to definitively identify paratuberculosis animals.

The average initial, maximum, and final ELISA values for the 3 experimental groups are depicted graphically in Figure 1, panel A. Results for cow 3056 were not included in the calculations because she is still alive and her maximum and final values are yet to be determined. The average initial ELISA values of the treated and nontreated positive groups were not significantly different $(P=0.39)$. The initial positive ELISA value obtained for each treated animal cannot 
Table 1. Johne's disease test parameters

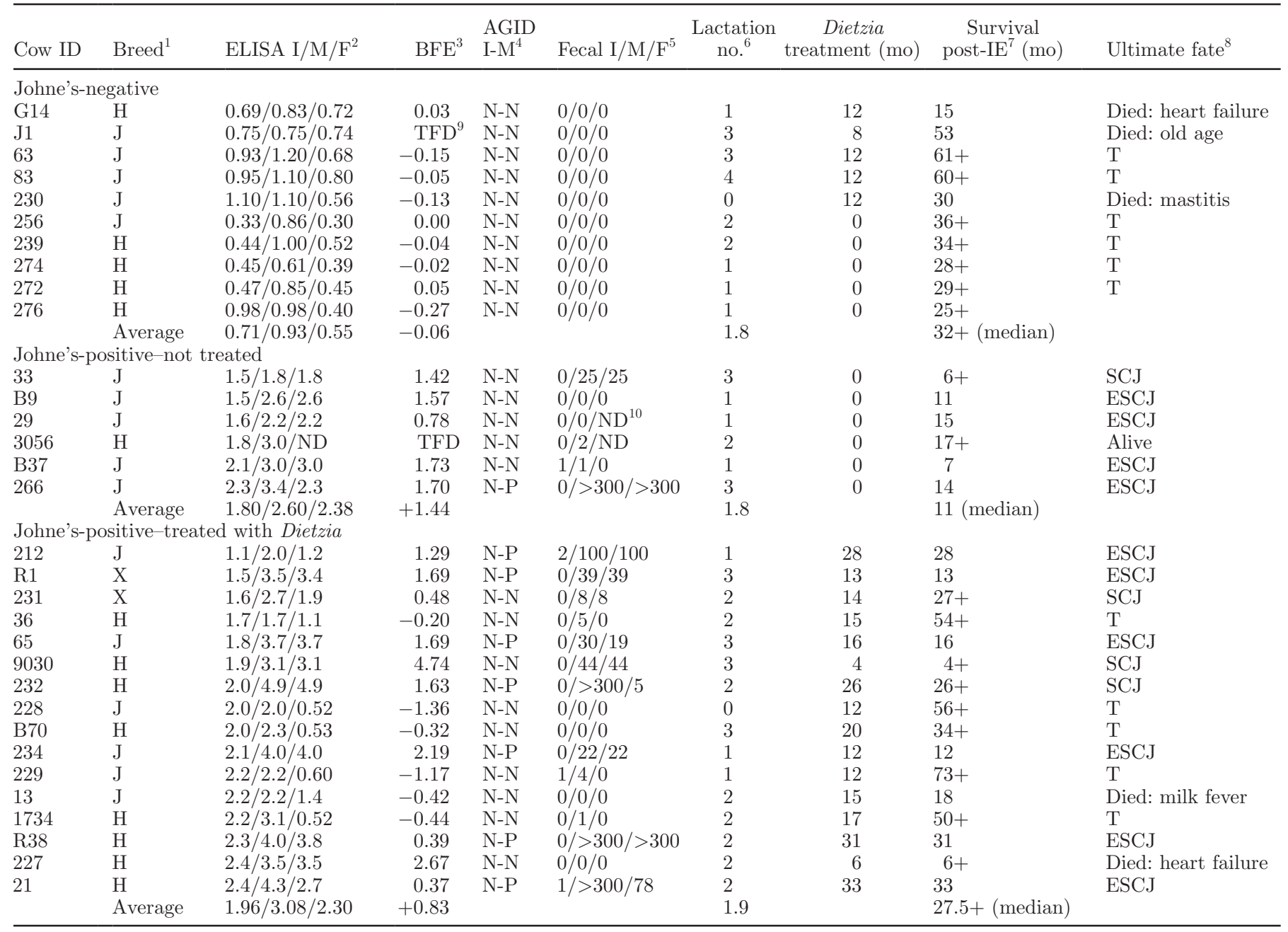

${ }^{1} \mathrm{~J}=$ Jersey, $\mathrm{H}=$ Holstein, $\mathrm{X}=$ cross.

${ }^{2}$ ELISA value: $\mathrm{I}=$ initial, $\mathrm{M}=$ maximum, $\mathrm{F}=$ final (within $4 \mathrm{wk}$ of demise).

${ }^{3} \mathrm{BFE}=$ best fit ELISA change/year.

${ }^{4}$ AGID (agar gel immunodiffusion) $=$ negative $(\mathrm{N})$-positive $(\mathrm{P}), \mathrm{I}=$ initial, $\mathrm{M}=$ maximum.

${ }^{5} \mathrm{Fecal}=\mathrm{cfu}$ of Mycobacterium avium ssp. paratuberculosis per $2 \mathrm{~g}, \mathrm{I}=$ initial, $\mathrm{M}=$ maximum, $\mathrm{F}=$ final (within 4 wk of demise).

${ }^{6}$ Lactation number at initial ELISA test.

${ }^{7} \mathrm{IE}=$ initial ELISA test shown in column 3.

${ }^{8} \mathrm{~T}=$ experiment terminated; SCJ = killed with subclinical Johne's disease; ESCJ = killed with end-stage clinical Johne's disease.

${ }^{9} \mathrm{TFD}=$ too few data $(2$ or less $)$.

${ }^{10} \mathrm{ND}=$ not done or yet to be done - data from cow 3056 not included in calculations.

be attributed to Dietzia because treatment was always started after an animal was detected positive. The averages for these 2 groups increased significantly from the initial to maximum values $(P=0.0003$ and $P=$ 0.0409 , respectively). This increase is highly unlikely to be caused by potential, cross-reactive antibodies induced by oral Dietzia sensitization because negative animals remained negative over their lifetime after prolonged oral treatment (Table 1). All of the average
ELISA values for the positive groups were significantly greater than the average values for the negative group $(P<0.0001)$. Negative animals showed minor positive or negative changes irrespective of whether they were treated or were not treated with Dietzia. None of these animals, over their lifetime, had an ELISA value $>1.4$, was ever fecal or AGID positive, or showed any sign of clinical disease. The 5 negative animals treated with Dietzia had an average yearly ELISA change of $-0.07 \pm$ 


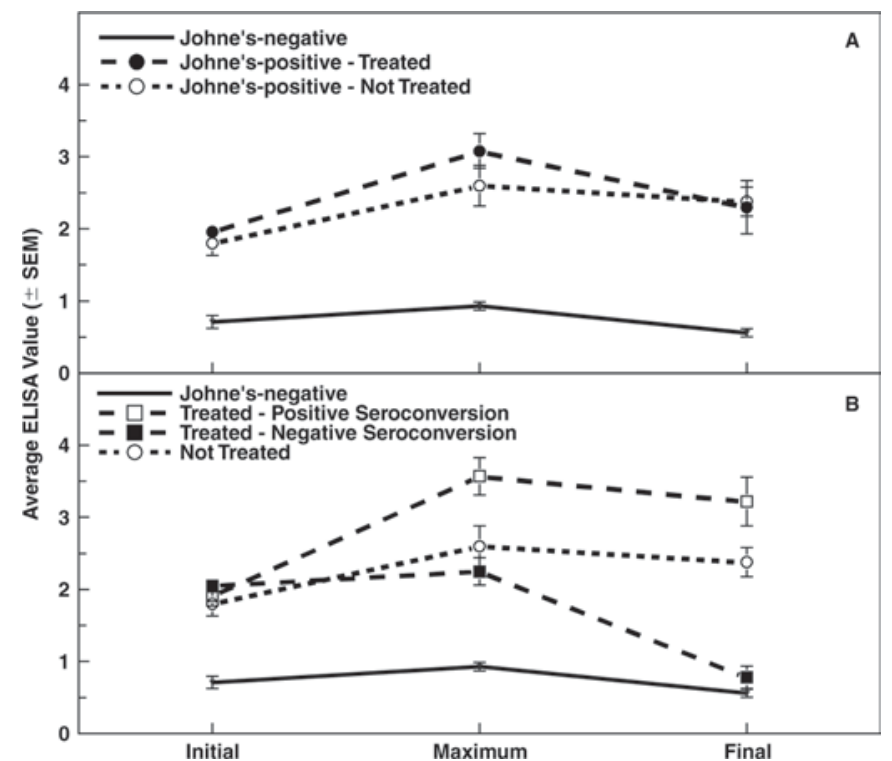

Figure 1. Average initial, maximum, and final ELISA values for Johne's-negative animals and for Johne's-positive treated and nontreated animals. Panel A shows the average ELISA values \pm SEM for each group; panel $\mathrm{B}$ shows the average ELISA values \pm SEM for the increasing and decreasing seroconversion subgroups.

$0.08 \mathrm{SD}$; the 5 not treated had an average yearly ELISA change of $-0.06 \pm 0.12 \mathrm{SD}$. There was no significant difference in the changes of these 2 groups $(P=0.81)$.

As shown in column 4 of Table 1, the treated group could be subdivided into 2 distinct groups based on increasing or decreasing longitudinal serology (our unpublished data). Of the 16 animals in this group, 10 had longitudinally increasing ELISA values and 6 had longitudinally decreasing ELISA values, indicating a loss of antibody. The treated subgroup with increasing changes had average initial, maximum, and final ELISA values similar to those of the nontreated group (Figure 1, panel B). The average initial, maximum, and final ELISA values for the treated subgroup with decreasing changes were dramatically different, with no significant change in average initial and maximum values $(P=$ $0.36)$ and a highly significant decrease of initial to final $(P=0.0001)$. Remarkably, although the average initial ELISA value of the treated subgroup with decreasing values was significantly higher than that of the negative group $(P<0.0001)$, there was no significant difference in the average final ELISA values of these 2 groups $(P$ $=0.13)$.

The longitudinal ELISA values of 6 positive animals that were at an early stage of disease and were not treated increased similarly to those reported by others (Waters et al., 2003; Nielsen and Toft, 2006), at an average yearly change of $+1.44 \pm 0.39$ SD. Four of these 6 died with end-stage clinical Johne's disease. Only cow
266 was AGID positive and only cows 29 and B9 did not shed MAP (see Table 1). The positive animals that were treated with Dietzia had an average yearly ELISA increase of $+0.83 \pm 1.59$. The average yearly change in ELISA values for the treated subgroup with positive changes was $+1.71 \pm 1.31$, which was significantly $(P$ $=0.0002$ ) greater than the average yearly decline of $-0.65 \pm 0.49$ for the negative changes group. The average change of the positive group was not significantly different from the average increase of the not treated positive group $(P=0.49)$. As shown in Table 1,9 of the 10 (except cow 227) treated positive animals with increasing ELISA values were shedding at the time of their demise. Also, 7 of these 9 became AGID positive and 6 were euthanized with end-stage clinical Johne's disease. Two of the end-stage diseased animals (212 and 234 ) were confirmed positive at autopsy. The 3 animals with increasing ELISA values that did not develop end-stage clinical disease were terminated because of non-Johne's disease health reasons.

In contrast to the 10 animals with increasing ELISA values, the 6 treated animals that had decreasing values all became ELISA negative $(\leq 1.4)$ and none became AGID positive. Although 3 of these animals (cows 36, 1734, and 229) were fecal positive early in their treatment (only once), all 6 were fecal negative for MAP at their demise. In addition, no pathological evidence or detection of MAP was found at autopsy of cow 13, who died early in the experiment because of milk fever complications postcalving. Thus, these 6 treated positive animals were considered cured. In no case did any of the cured animals show any sign of disease for the remainder of their lives, ranging from 18 to 73 mo after the first detected positive ELISA. Whether "cured" means these animals were actually MAP-free or that MAP was simply reduced to undetectable fecal levels and to sub-threshold levels incapable of stimulating antibody synthesis is extremely difficult to discern. Either alternative would be advantageous for controlling the spread of MAP via fecal shedding.

The survival times of the different experimental groups were analyzed using the Kaplan-Meier method to estimate survival probabilities, which takes into account animals that did not succumb to Johne's disease (such as those cured) but were terminated for other reasons. Figure 2 depicts this survival analysis for the negative animals, the treated positive animals, and the nontreated positive animals. The survival of the positive group that was treated with Dietzia was not significantly different from the survival of the negative group $(P=0.22)$. In addition, the survival of the treated group was significantly greater than the survival of the nontreated group $(P=0.0116)$. The median survival time of the nontreated positive group was less than 


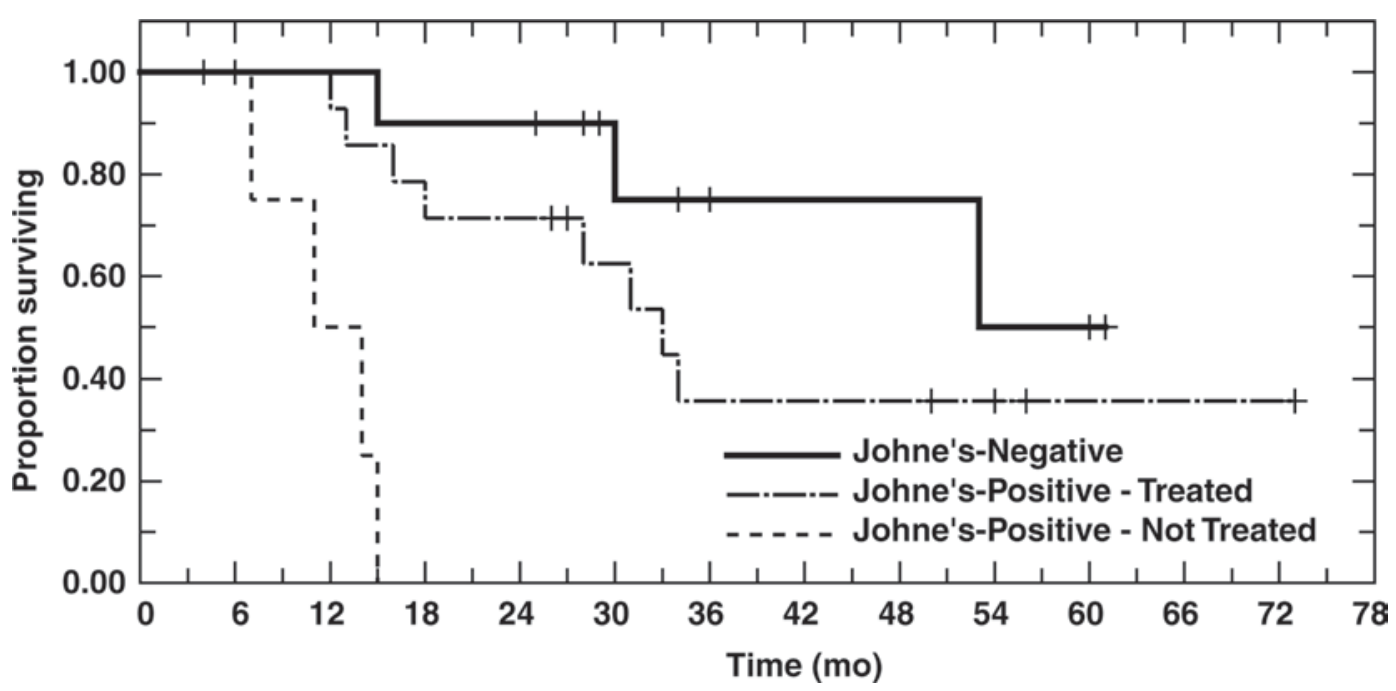

Figure 2. Kaplan-Meier survival graph for Johne's-negative animals and for Johne's-positive treated and nontreated animals. Many Johne'snegative and Johne's-positive animals terminated for non-Johne's insults are shown by the tick marks on each curve.

expected based on the literature, in which Holsteins were the predominant breed studied. This may be because 5 of the 6 were Jerseys or because of the small number of animals in the group. In the treated group the median survival times were 20 mo for crosses, 23 mo for Jerseys, and 32 mo for Holsteins; however, the latter 2 durations were not significantly different $(P=$ $0.88)$. Even though $37.5 \%$ of the animals in the treated group were cured, this difference was not statistically significant $(P=0.15)$ from the absence of any cures in the small number of animals in the nontreated group. As alluded to earlier, this group was small because it was not our goal to confirm the results of others that all positive animals, not treated, would succumb to their disease. If more animals had been included in the nontreated group, statistical significance would have been likely. Indeed, 10 of 13 nontreated adult cows (with ELISA values $>2.5$ ) that did not survive sufficiently to derive longitudinal analyses succumbed with end-stage disease (our unpublished data).

In summary, an oral, daily probiotic treatment of Dietzia effectively increased the survival of cows with early-stage Johne's disease and in certain cases cured the animal. However, some intriguing questions remain to be investigated: Would a similar percentage of animals be cured if treatment were initiated at a more advanced stage of disease? Why did not all animals benefit from the Dietzia treatment?

\section{ACKNOWLEDGMENTS}

This research was funded, in part, by NIH grant R01AI027331, by Altick Associates (River Falls, WI), and by Paralab LLC (Eau Claire, WI). We thank
William D. Richards (retired; Allied Research, Grand Junction, CO) for the initial ICON 6 isolate (Dietzia).

\section{REFERENCES}

Abubakar, I., D. Myhill, S. H. Aliyu, and P. R. Hunter. 2008. Detection of Mycobacterium avium subspecies paratuberculosis from patients with Crohn's disease using nucleic acid-based techniques: A systematic review and meta-analysis. Inflamm. Bowel Dis. 14:401-410.

Abubakar, I., D. J. Myhill, A. R. Hart, I. R. Lake, I. Harvey, J. M. Rhodes, R. Robinson, A. J. Lobo, C. S. Probert, and P. R. Hunter. 2007. A case-control study of drinking water and dairy products in Crohn's disease-Further investigation of the possible role of Mycobacterium avium paratuberculosis. Am. J. Epidemiol. 165:776-783.

Antognoli, M. C., F. B. Garry, H. L. Hirst, J. E. Lombard, M. M. Dennis, D. H. Gould, and M. D. Salman. 2008. Characterization of Mycobacterium avium subspecies para-tuberculosis disseminated infection in dairy cattle and its association with antemortem test results. Vet. Microbiol. 127:300-308.

Ayele, W. Y., P. Svastova, P. Roubal, M. Bartos, and I. Pavlik. 2005 Mycobacterium avium subspecies paratuberculosis cultured from locally and commercially pasteurized cows' milk in the Czech Republic. Appl. Environ. Microbiol. 71:1210-1214.

Behr, M. A., and V. Kapur. 2008. The evidence for Mycobacterium paratuberculosis in Crohn's disease. Curr. Opin. Gastroenterol. 24:17-21.

Benedictus, A., R. M. Mitchell, M. Linde-Widmann, R. Sweeney, T. Fyock, Y. H. Schukken, and R. H. Whitlock. 2008. Transmission parameters of Mycobacterium avium subspecies paratuberculosis infections in a dairy herd going through a control program. Prev. Vet. Med. 83:215-227.

Chamberlin, W., D. Y. Graham, K. Hulten, H. M. El-Zimaity, M. R. Schwartz, S. Naser, I. Shafran, and F. A. El-Zaatari. 2001. Review article: Mycobacterium avium subsp. paratuberculosis as one cause of Crohn's disease. Aliment. Pharmacol. Ther. 15:337-346.

Chamberlin, W. M., and S. A. Naser. 2006. Integrating theories of the etiology of Crohn's disease. On the etiology of Crohn's disease; questioning the hypotheses. Med. Sci. Monit. 12:27-33.

Chiodini, R. J. 1989. Crohn's disease and the mycobacterioses: A review and comparison of two disease entities. Clin. Microbiol. Rev. 2:90-117. 
Chiodini, R. J., H. J. Van Kruiningen, and R. S. Merkal. 1984. Ruminant paratuberculosis (Johne's disease)-The current status and future prospects. Cornell Vet. 74:218-262.

Clarke, C. J. 1997. The pathology and pathogenesis of paratuberculosis in ruminants and other species. J. Comp. Pathol. 116:217-261.

Ellingson, J. L. E., J. L. Anderson, J. J. Koziczkowski, R. P. Radcliff, S. J. Sloan, S. E. Allen, and N. M. Sullivan. 2005. Detection of viable Mycobacterium avium subs. paratuberculosis in retail pasteurized whole milk by two culture methods and PCR. J. Food Prot. 68:966-972.

Feller, M., K. Huwiler, R. Stephan, E. Altpeter, A. Shang, H. Furrer, G. E. Pfyffer, T. Jemmi, A. Baumgartner, and M. Egger. 2007. Mycobacterium avium subspecies paratuberculosis and Crohn's disease: A systematic review and meta-analysis. Lancet Infect. Dis. 7:607-613.

Grant, I. R., H. J. Ball, and M. T. Rowe. 2002. Incidence of Mycobacterium avium subsp. paratuberculosis in bulk raw and commercially pasteurized cow's milk from approved dairy processing establishments in the United Kingdom. Appl. Environ. Microbiol. 68:2428-2435.

Harris, J. E., and A. M. Lammerding. 2001. Crohn's disease and Mycobacterium avium subspecies paratuberculosis: Current issues. J. Food Prot. 64:2103-2110.

Hermon-Taylor, J., and T. Bull. 2002. Crohn's disease caused by Mycobacterium avium subspecies paratuberculosis: A public health tragedy whose resolution is long overdue. J. Med. Microbiol. $51: 3-6$.

Hermon-Taylor, J., T. J. Bull, J. M. Sheridan, J. Cheng, M. L. Stellakis, and N. Sumar. 2000. Causation of Crohn's disease by Mycobacterium avium subspecies paratuberculosis. Can. J. Gastroenterol. 14:521-539.

Kennedy, D. J., and G. Benedictus. 2001. Control of Mycobacterium avium subsp. para-tuberculosis infection in agricultural species. Rev. Sci. Tech. 20:151-179.

Lu, Z., R. M. Mitchell, R. L. Smith, J. S. Van Kessel, P. P. Chapagain, Y. H. Schukken, and Y. T. Grohn. 2008. The importance of culling in Johne's disease control. J. Theor. Biol. 254:135-146.

McDowell, R. M., and M. D. McElvaine. 1997. Long-term sequelae to foodborne disease. Rev. Sci. Tech. 16:337-341.

McKenna, S. L., G. P. Keefe, A. Tiwari, J. VanLeeuwen, and H. W. Barkema. 2006. Johne's disease in Canada part II: Disease impacts, risk factors, and control programs for dairy producers. Can. Vet. J. 47:1089-1099.

Nielsen, S. S., Y. T. Grohn, and C. Enevoldsen. 2002. Variation of the milk antibody response to paratuberculosis in naturally infected dairy cows. J. Dairy Sci. 85:2795-2802.

Nielsen, S. S., and N. Toft. 2006. Age-specific characteristics of ELISA and fecal culture for purpose-specific testing for paratuberculosis. J. Dairy Sci. 89:569-579.

Pickup, R. W., G. Rhodes, T. L. Bull, S. Arnott, K. Sidi-Boumedine, M. Hurley, and J. Hermon-Taylor. 2006. Mycobacterium avium subsp. paratuberculosis in lake catchments, in river water abstracted for domestic use, and in effluent from domestic sewage treatment works: diverse opportunities for environmental cycling and human exposure. Appl. Environ. Microbiol. 72:4067-4077.
Richards, W. D. 1988. In vitro and in vivo inhibition of Mycobacterium paratuberculosis by iron deprivation: A hypothesis. Pages 87-94 in Proc. Conf. Johne's Disease, Australia. A. R. Milner and P. R. Wood, ed. International Association of Paratuberculosis Inc., Rehoboth, MA.

Scanu, A. M., T. J. Bull, S. Cannas, J. D. Sanderson, L. A. Sechi, G. Dettori, S. Zanetti, and J. Hermon-Taylor. 2007. Mycobacterium avium subsp. paratuberculosis infection in cases of irritable bowl syndrome and comparison with Crohn's disease and Johne's disease: Common neural and immune pathogenicities. Clin. Microbiol. 45:3883-3890.

Seitz, S. E., L. E. Heider, W. D. Heuston, S. Bech-Nielsen, D. M. Riggs, and L. Spangler. 1989. Bovine fetal infection with Mycobacterium paratuberculosis. J. Am. Vet. Med. Assoc. 194:1423-1426.

Sherman, D. M., R. J. Markham, and F. Bates. 1995. Agar gel immunodiffusion test for diagnosis of clinical paratuberculosis in cattle. J. Am. Vet. Med. Assoc. 185:179-182.

Streeter, R. N., G. F. Hoffsis, S. Cech-Nielsen, W. P. Shulaw, and D. M. Rings. 1995. Isolation of Mycobacterium paratuberculosis from colostrum and milk of subclinically infected cows. Am. J. Vet. Res. 56:1322-1324.

Sweeney, R. W. 1996. Transmission of paratuberculosis. Vet. Clin. North Am. Food Anim. Pract. 12:305-312.

Sweeney, R. W., R. H. Whitlock, and A. E. Rosenberger. 1992a. Mycobacterium paratuberculosis isolated from fetuses of infected cows not manifesting signs of the disease. Am. J. Vet. Res. $53: 477-480$

Sweeney, R. W., R. H. Whitlock, and A. E. Rosenberger. 1992b. Mycobacterium paratuberculosis cultured from milk and supramammary lymph nodes of infected asymptomatic cows. J. Clin. Microbiol. 30:166-171.

Tavornpanich, S., W. O. Johnson, R. J. Anderson, and I. A Gardner. 2008. Herd characteristics and management practices associated with seroprevalence of Mycobacterium avium subspecies paratuberculosis infection in dairy herds. Am. J. Vet. Res. 69:904911.

Taylor, T. K., C. R. Wilks, and D. S. McQueen. 1981. Isolation of Mycobacterium para-tuberculosis from the milk of a cow with Johne's disease. Vet. Rec. 109:532-533.

Waters, W. R., J. M. Miller, M. V. Palmer, J. R. Stabel, D. E. Jones, K. A. Koistinen, E. M. Steadham, M. J. Hamilton, W. C. Davis, and J. P. Bannantine. 2003. Early induction of humoral and cellular immune responses during experimental Mycobacterium avium subsp. paratuberculosis infection of calves. Infect. Immun. 71:5130-5138.

Wells, S. J., and B. A. Wagner. 2000. Herd-level risk factors for infection with Mycobacterium paratuberculosis in US dairies and association between familiarity of the herd manager with the disease or prior diagnosis of the disease in that herd and use of preventive measures. J. Am. Vet. Med. Assoc. 216:1450-1457.

Whittington, R. J., and P. A. Windsor. 2009. In utero infection of cattle with Mycobacterium avium subsp. paratuberculosis: A critical review and meta-analysis. Vet. J. 179:60-69.

Woese, C. R. 1987. Bacterial evolution. Microbiol. Rev. 51:221-271. 